\title{
BMJ Open Impact of particulate matter on mothers and babies in Antwerp (IPANEMA): a prospective cohort study on the impact of pollutants and particulate matter in pregnancy
}

\author{
Lena Van den Eeden, ${ }^{1,2}$ Nathalie Lambrechts, ${ }^{3}$ Veerle Verheyen, ${ }^{3,4}$ Mario Berth, ${ }^{5}$ \\ Greet Schoeters, ${ }^{3,4,6}$ Yves Jacquemyn ${ }^{2,7}$
}

To cite: Van den Eeden L, Lambrechts $\mathrm{N}$, Verheyen $\mathrm{V}$, et al. Impact of particulate matter on mothers and babies in Antwerp (IPANEMA): a prospective cohort study on the impact of pollutants and particulate matter in pregnancy. BMJ Open 2018;8:e20028. doi:10.1136/ bmjopen-2017-020028

\section{- Prepublication history for} this paper is available online. To view these files, please visit the journal online (http://dx.doi. org/10.1136/bmjopen-2017020028).

Received 10 0ctober 2017 Revised 24 November 2017 Accepted 6 February 2018

\section{Check for updates}

${ }^{1}$ Thomas More University College, Lier, Belgium

${ }^{2}$ Faculty of Medicine and Health Sciences, University of Antwerp, Wilrijk, UA, Belgium

${ }^{3} \mathrm{VITO}$, Boeretang, Mol, Belgium ${ }^{4}$ Department of Biomedical Sciences, University of Antwerp, Wilrijk, Belgium

${ }^{5}$ Algemeen Medisch

Laboratorium, Antwerp, Belgium

${ }^{6}$ University of Southern Denmark, Odense, Denmark ${ }^{7}$ Departement of Gynaecology and Obstetrics, Antwerp University Hospital, Edegem, Belgium

Correspondence to Lena Van den Eeden; leen.vandeneeden@ thomasmore.be

\section{ABSTRACT}

Introduction Air pollution is a hot topic and is known to cause multiple health issues. Especially pregnant women seem to be vulnerable to environmental issues. There are data suggesting that exposure contributes to hypertensive disorders. This study aims to evaluate the effects of exposure to particulate matter (PM) and outdoor air pollutants on the clinical pregnancy outcome for mother and child and to determine which biochemical changes in maternal, placental and cord blood best explain this effect. Methods and analysis This study is a prospective cohort study. We aim to recruit 200 pregnant women. The outcome measurements will include maternal parameters, labour parameters and neonatal parameters. Multiple samples will be analysed such as maternal urine samples (8-oxo-deoxyguanosine), maternal blood samples (routine blood sampling, biomarkers of pre-eclampsia and transcript markers), maternal hair samples, neonatal blood samples (transcript markers) combined with extensive questionnaires.

Ethics and dissemination We obtain informed consent from each participant prior to enrolment in the study. The study has received approval by the Ethical Committee of the Antwerp University Hospital (14/40/411). IPANEMA is the first prospective study to assess the impact of PM on mothers and babies in Antwerp, Belgium. Findings from this study will contribute to improve knowledge on the impact of exposure to air pollution on mothers and babies and will also define biomarkers as predictors for pregnant women at risk.

Trial registration ClinicalTrials.gov: 14/40/411.

Registered 22-10-2015.

\section{INTRODUCTION}

An emerging body of evidence indicates that there is an association between air pollution exposure in pregnancy and adverse pregnancy outcomes. ${ }^{12}$ Most studies have estimated personal exposure to air pollution by modelling data from outdoor monitoring stations and interpolating them to the home address of the study participants. The exposure data
Strengths and limitations of this study

- First prospective study to assess the impact of particulate matter on mothers and babies in Antwerp, Belgium.

- Large questionnaires to minimalise bias from multiple factors.

- Only eligible for Dutch speaking women, which gives a bias.

are averaged over different time periods and in general time spent in traffic or exposure at the workplace or indoors are not taken into account.

Particulate matter (PM) is an important component of outdoor air pollution. PM has different sizes, but especially fine PM, with an aerodynamic diameter less than $2.5 \mu \mathrm{m}$ $\left(\mathrm{PM}_{25}\right)$ is of great interest, because of its small size, large specific surface area and long residence in air, and thus is more likely to adsorb harmful substances. $\mathrm{PM}_{2.5}$ consists of a mixture of solid and liquid particles, emitted from a variety of sources. Because of its size it can penetrate in airways until the alveoli from where the particles or the absorbed pollutants may be translocated into the bloodstream. ${ }^{3}$

A significant positive association between exposure to fine PM during the third trimester and pre-eclampsia has been observed in a study by Dadvand et al among over 8000 pregnant women in Barcelona, Spain. ${ }^{2}$

Exposure to local traffic-generated air pollution during pregnancy is also known to increase the risk of pre-eclampsia and preterm birth. ${ }^{4}$ Proximity to major roads is associated with an increased risk of pre-eclampsia, but not with a higher risk of gestational diabetes, placental abruption or placenta praevia. ${ }^{5}$ 
Hypertensive disorders of pregnancy affect about $10 \%$ of all pregnant women. They are an important cause of severe morbidity and even mortality. ${ }^{6}$ Pre-eclampsia complicates $2 \%-8 \%$ of pregnancies and is a major contributor to maternal and neonatal mortality worldwide. ${ }^{7}$

Pre-eclampsia is generally defined as de novo hypertension occurring after 20 weeks of pregnancy (with systolic blood pressure $\geq 140 \mathrm{~mm} \mathrm{Hg}$ and/or diastolic blood pressure $\geq 90 \mathrm{~mm} \mathrm{Hg}$ ) and proteinuria ( $\geq 300 \mathrm{mg}$ in 24 hours) ${ }^{6-8}$

The cause of pre-eclampsia still remains largely unknown, but a disturbed placental function early in pregnancy has been suggested as a specific underlying mechanism. In normal pregnancy the uteroplacental arteries undergo a series of pregnancy-related changes in the first 20 weeks. Invasive trophoblasts replace endothelial cells and smooth muscle cells in the media, causing the arteries to lose elasticity and to dilate to wide tubes, without the ability to contract effectively. They also lose their vasomotor control. This mechanism occurs in order to guarantee maternal blood supply to the placenta: the loss of maternal blood flow resistance and the increase of uteroplacental perfusion meet the requirements of the fetus. ${ }^{9}$ In addition, circulating antiangiogenic factors may play a role in the pathogenesis. The transmembrane protein vascular endothelial growth factor (VEGF) receptor fms-like tyrosine kinase 1 (Flt-1) binds with high affinity to VEGF and to placental growth factor (PIGF). ${ }^{10}$ Flt-1 is involved in normal angiogenesis. Soluble Flt-1 (sFlt-1) is a variant of Flt-1 that lacks a transmembrane protein, and is a naturally occurring antagonist of VEGF and PlGF. Production of sFlt-1 appears to be expressed by endothelial cells and trophoblasts in response to reduced oxygen tensions. ${ }^{1112}$ In developing pre-eclamptic placenta the normal process of remodelling of the uterine spiral arteries is impaired, resulting in reduced perfusion, increased oxidative stress and inflammation. ${ }^{12}$

Although there are yet no validated biomarkers that allow to identify women at risk for pre-eclampsia, levels of angiogenic and antiangiogenic factors are altered in women with pre-eclampsia. Levels of sFlt-1 are elevated and levels of PIGF are decreased, even before clinical symptoms of disease were overt. ${ }^{1314}$ The ratio of sFlt- $1 /$ PlGF is a promising set of biochemical markers for prediction of pre-eclampsia. There is a pathogenic difference between early pre-eclampsia (onset of disease before 32-34 weeks of pregnancy) and late pre-eclampsia (onset of disease after 32-34 weeks of pregnancy). Early pre-eclampsia is characterised by a significant placental dysfunction, leading to a high risk of intrauterine growth restriction. In late pre-eclampsia there is more evidence of pre-existing maternal inflammation and/or cardiovascular maladaptation. ${ }^{715}$ The distinction is not always that clear, and is under debate.

Cystatin C, a protease inhibitor, is also increased in pre-eclamptic women. Cystatin $\mathrm{C}$ can be used as an endogenous marker for renal function, synthesised by all nucleated cells, at a constant rate and exclusively eliminated by glomerular filtration. ${ }^{16}$ Saleh et al found that levels of cystatin $\mathrm{C}$ were significantly higher in the second trimester of pregnancy in women who developed pre-eclampsia. ${ }^{17}$ Angiogenesis-related and stress-related pathways may underlie the vascular effects of $\mathrm{PM}_{2.5}$ exposure during pregnancy. ${ }^{10} 1819$ Endothelin-1 is an established biomarker of endothelium dysfunction. ${ }^{20}$ Nuclear factor of activated T cells (NFAT) contributes to diverse functions and was identified as a critical component of VEGF-induced angiogenesis. ${ }^{21}$

Higher exposure to $\mathrm{PM}_{2.5}$ and $\mathrm{O}_{3}$ during the first trimester are associated with pre-eclampsia, gestational hypertension and preterm delivery. ${ }^{22}$

We hypothesise that exposure to $\mathrm{PM}_{2.5}$ in the first trimester of pregnancy interferes with the maternal vascular remodelling process and that impaired remodelling between 9 weeks and 18 weeks results in reduced placental perfusion and oxidative stress. ${ }^{23}$

In 2011 WHO stated in a report that supplementation of calcium during pregnancy for women at high risk of developing pre-eclampsia is recommended. ${ }^{6}$ They also state that low-dose acetylsalicylic acid $(75 \mathrm{mg} /$ day) is recommended in the same population. A recent study by Souza et $a l^{24}$ confirmed that supplementation with $100 \mathrm{mg}$ aspirin plus $2 \mathrm{~g}$ calcium when started after 20 weeks of gestational age reduced the rate of superimposed pre-eclampsia and intrauterine growth restriction in women with chronic hypertension and abnormal second-trimester uterine artery Doppler. ${ }^{24}$ If this study can predict which women are at risk for hypertensive disorders during pregnancy or pre-eclampsia, supplementation of aspirin and calcium can be started in order to reduce perinatal risks.

\section{Objectives and aims of the IPANEMA Study}

This study aims to evaluate the effects of exposure to PM and outdoor air pollutants on the clinical pregnancy outcome for mother and child and to determine which biochemical changes in maternal, placental and cord blood best explain this effect.

- Objective 1: Correlate estimated personal $\mathrm{PM}_{2.5}$ exposures to angiogenesis-related markers for hypertensive disorders in pregnant woman.

- Objective 2: Gain more insight in the pathophysiology behind cardiovascular effects of air pollution during pregnancy, plausible underlying molecular pathways will be assessed, for example, stress-related and angiogenesis-related pathways.

\section{METHODS AND ANALYSIS}

\section{Study design and setting}

The IPANEMA Study is a regional prospective cohort study, recruiting pregnant women in the Antwerp region in Belgium.

Data are collected during pregnancy and at delivery, as well as immediately postpartum. Blood samples, hair samples and anthropometric measurements are obtained. Self-administered questionnaires are completed at 


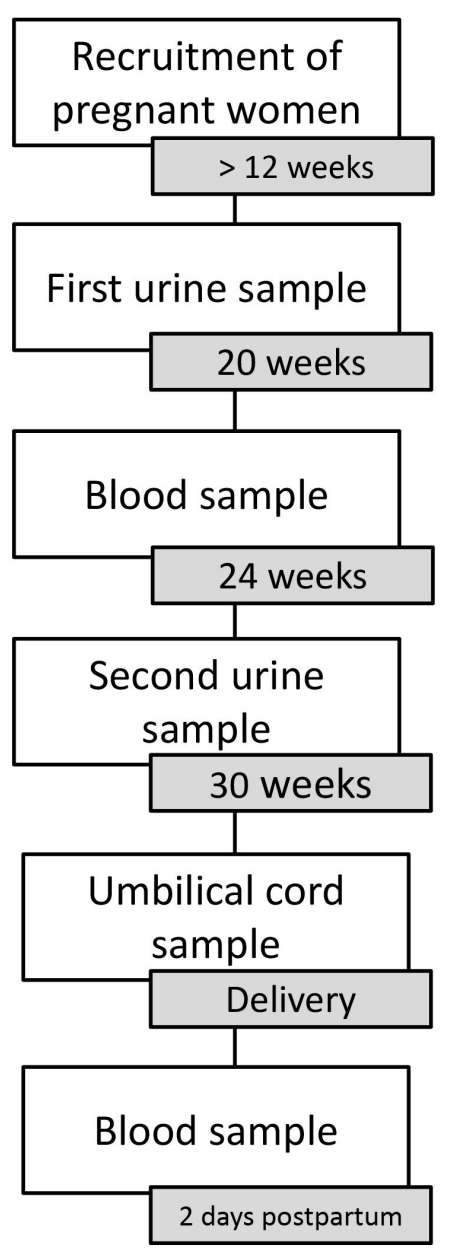

Figure 1 Visits and interventions during the IPANEMA Study.

different time frames in pregnancy, in relation to sample taking (see figure 1).

\section{Study sample}

Study cohort

Pregnant women are recruited within the network of Antwerp University. The leading centre will be University Hospital Antwerp, a tertiary centre with a maternal intensive care unit and a neonatal intensive care unit and 1000 deliveries a year.

The aim is to enrol 200 pregnant women over a 3-year recruitment period. An increase of $2 \%$ of pregnant women who develop pre-eclampsia or hypertensive disorders can be considered clinically relevant.

Diagnosis of pre-eclampsia (onset of a new episode of hypertension during pregnancy (with persistent diastolic blood pressure $>90 \mathrm{~mm} \mathrm{Hg}$ ) with the occurrence of substantial proteinuria (>0.3 g/24hours) ) and hypertensive disorders are made using WHO guidelines. ${ }^{6}$

\section{Eligibility criteria}

The inclusion criteria are (see figure 2)

- A singleton pregnancy

- More than 12 weeks of gestational age

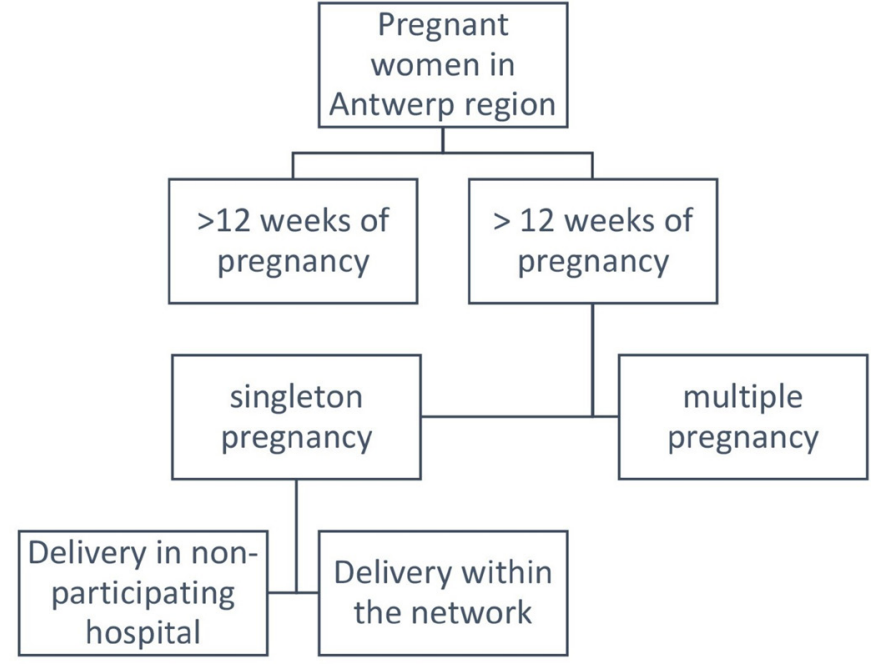

Figure 2 Eligibility criteria.

- Delivery planned in a hospital within the network of Antwerp University

- Dutch speaking

The exclusion criteria are:

- Multiple pregnancy

- Planned home delivery or planned delivery in another hospital

Power calculation and statistical analyses

The incidence of pre-eclampsia in pregnancy is approximately $5 \%$. An increase of $2 \%$ is of interest. A doubling in incidence is therefore clinically relevant. With a sample size of 200 subjects, a doubling from $5 \%$ to $10 \%$ can be detected with $80 \%$ power and $5 \%$ significance level.

Data analysis will be done using SPSS V.24.0.

Recruitment started in the summer of 2015 and we aim to collect all participants by the end of 2018 .

\section{Study visits}

Women are recruited by the midwife or obstetrician at 12 weeks of gestational age, typically at the second routine antenatal visit.

Signed informed consent for participating in the study is obtained before the start.

Blood collections (maternal and umbilical cord)

Venous blood is drawn around 24 weeks of gestational age (table 1).

Umbilical cord blood is taken at the time of birth (table 2).

Measurement of molecular pathway and transcript markers For analysis of the m(i) RNA profile, whole blood is collected in Tempus Blood RNA tubes (Applied Biosystems) and stored at $-80^{\circ} \mathrm{C}$ until analysis. Telomere length and DNA methylation will be assessed on blood samples collected in EDTA tubes, which are stored at $-80^{\circ} \mathrm{C}$.

In order to gain more insight into pathophysiological pathways, relevant biomarkers and transcript markers will be analysed. Specific transcript markers will be selected that were previously associated with exposure 
to PM, intrauterine growth retardation or stress-induced and angiogenesis pathways, for example, glucocorticoid receptor signalling pathway, NFAT signalling pathway, and so on. ${ }^{25}$

\section{Urine collections}

Urine samples are taken by the pregnant women themselves, after a minimum of 8 hours fasting (morning urine). Samples are handed to the midwife at the antenatal visit, and placed in a freezer at $-20^{\circ} \mathrm{C}$ within 12 hours of collection. Analysis will be performed at the Flemish Institute for Technological Research.

Two urine samples are collected at 20 weeks and at 30 weeks gestational age; 8-oxo-deoxyguanosine will be determined in the samples.

\section{Hair collections}

Being incorporated into the growing hair, hair cortisol concentrations (HCCs) provide a retrospective reflection of integrated cortisol secretion over periods of several months. ${ }^{26}$

Hair samples are drawn by the midwife around 24 weeks of gestational age and 3 days after delivery. Long periods of stress are associated with increased HCCs. ${ }^{26}$ Confounding variables are low maternal education, season of delivery, smoking during pregnancy and obesity. ${ }^{27}$ Titanium scissors are cleaned with denaturated ethanol and the midwife wears disposable gloves in order to limit contamination. A lock of hair with a thickness of a match $(2 \mathrm{~mm})$ and a length of $4 \mathrm{~cm}$ will be taken and put in an envelope. Analysis will be performed at the University of Southern Denmark.

\section{Statistical analysis}

Regression models will be calculated. In particular, birth weight will be correlated with $\mathrm{PM}_{2.5}$ values using linear

\section{Table 1 Tests on maternal blood sample}

Routine blood sampling Urea, creatinin, CRP, LDH, AST, (UZA)

ALT, uric acid, APTT, PT, fibrinogen, D-dimers, glucose, erythrocytes/ haematocrit, haemoglobin, thrombocytes, leucocytes, ferritin, toxoplasmosa (lgG and $\lg M)$, Cytomegalovirus (IgG and IgM), herpes simplex IgG, Varicella zoster IgG, rubella IgG, parvovirus (IgG and IgM), Syphilis (RPR and TPHA), Indirect Coomb's test

\begin{tabular}{ll}
$\begin{array}{l}\text { Biomarkers of pre- } \\
\text { eclampsia }\end{array}$ & sFlt-1, PIGF, cystatin C, endothelin \\
$\begin{array}{ll}\text { Molecular pathway and } \\
\text { transcript markers }\end{array}$ & $\begin{array}{l}\text { m(i)RNA expression, telomere } \\
\text { length, DNA methylation }\end{array}$ \\
\hline
\end{tabular}

ALT, alanine transaminase; APTT, activated partial thromboplastin time; AST, aspartate aminotransferase; CRP, C-reactive protein; LDH, lactate dehydrogenase; PIGF, placental growth factor; PT, prothrombin time; RPR, rapid plasma reagin; sFlt-1, soluble fms-like tyrosine kinase 1; TPHA, Treponema pallidum haemagglutination; UZA, University Hospital Antwerp.
Table 2 Tests on umbilical blood sample

Transcript markers m(i)RNA expression, telomere length, DNA methylation

regression, pre-eclampsia with blood pressure evolution and $\mathrm{PM}_{2.5}$ using logistic regression, birth weight, birth weight (dichotomised) and pre-eclampsia with sFlt-1, PlGF, cystatin $\mathrm{C}$ and $\mathrm{PM}_{2.5}$ using linear and logistic regressions, respectively, and birth weight (dichotomised) versus inflammatory parameters using logistic regression. The effect of the following a priori covariates will be analysed: history of pre-eclampsia, diabetes, BMI at start, age, smoking, intake of low dose aspirin and pre-existing hypertension. These factors will however not be a reason for exclusion.

\section{Questionnaires}

There are four different questionnaires:

- Questionnaire on general habits, socioeconomic factors, lifestyle and eating habits

We use a self-designed questionnaire to extensively collect information on ethnic origin and education level, on employment, income and work environment, on family history of diseases and chronic disorders, on eating habits, on previous pregnancies and other habits (smoking, alcohol consumption).

- Questionnaire on lifestyle during previous 3 days

We use a self-designed questionnaire to extensively collect information on contact with possible toxic factors and on eating pattern of the previous 3 days.

- Questionnaire on residential facts

We use a self-designed questionnaire to collect information about the exact location of the bedroom window and other possible factors influencing concentrations of PM.

- Questionnaire on stress factors and birth facts

We use a self-designed questionnaire to extensively collect information on stress levels during pregnancy, on birth facts and on medication during pregnancy.

Questionnaires will be taken at different visits (table 3)

Additional clinical information and data about medication will be obtained by detailed data extraction from the hospital records.

\section{Outcomes of the study}

\section{Diagnosis of cardiovascular disorders}

Pre-eclampsia is defined as the onset of a new episode of hypertension during pregnancy (with persistent diastolic blood pressure $>90 \mathrm{~mm} \mathrm{Hg}$ ) with the occurrence of substantial proteinuria ( $>0.3 \mathrm{~g} / 24$ hours).

\section{Pregnancy and delivery outcome data}

Maternal data that are prospectively collected are: pregnancy duration, pre-eclampsia, hypertensive disorders.

Delivery data are: type of labour, type of delivery. 


\begin{tabular}{|c|c|c|c|c|}
\hline & $<12$ weeks & 20 weeks & 30 weeks & birth \\
\hline $\begin{array}{l}\text { Questionnaire on general habits, } \\
\text { socioeconomic factors, lifestyle and } \\
\text { eating habits }\end{array}$ & Recruitment & & & \\
\hline $\begin{array}{l}\text { Questionnaire on lifestyle during } \\
\text { previous } 3 \text { days }\end{array}$ & & Urine sample 1 & Urine sample 2 & \\
\hline Questionnaire on residential facts & & & $4-8$ weeks prior to visit & \\
\hline
\end{tabular}

Neonatal data are: birth weight, preterm delivery, Apgar Scores at $1 \mathrm{~min}, 5 \mathrm{~min}$ and $10 \mathrm{~min}$, congenital anomalies, and so on.

\section{Other study measurements}

\section{Measurement of sFIt-1, PIGF and cystatin C}

Blood samples taken at the study visit around 24 weeks of gestational age for the analyses of sFlt-1, PlGF and cystatin $\mathrm{C}$ are collected, processed and aliquoted within 24 hours of collection. The serum samples are stored at $-80^{\circ} \mathrm{C}$ until analysis at Algemeen Medisch Laboratorium. sFlt-1 and PIGF will be determined using an electrochemiluminescence-based sandwich immunoassay on Cobas e 411 (Roche Diagnostics, Mannheim, Germany). ${ }^{28}{ }^{29}$ Cystatin $\mathrm{C}$ will be determined using a particle-enhanced immunonephelometric assay (N Latex cystatin C, Siemens Healthcare Diagnostics, Marburg, Germany) by use of a BN II nephelometer (Siemens Healthcare Diagnostics). This assay has a calibration traceable to the first certified reference material for cystatin $\mathrm{C}$ in human serum (ERM-DA471/IFCG). ${ }^{30}$

\section{DISCUSSION}

IPANEMA is the first prospective study to assess the impact of PM on mothers and babies in Antwerp, Belgium.

Exposure to PM is debated on the political scene but robust data showing a relationship with hypertensive disorders of pregnancy are difficult to find. In Antwerp the design of a safe and functional tunnel for the highway is now focus of public discussion. Following the Barcelona experience a group of people want an urban tunnel complex to lower the amount of PM.

IPANEMA would like to create robust data on the correct impact of air pollution, in particular on pregnant women, a vulnerable population. IPANEMA uses prospective data from the beginning of the pregnancy till childbirth.

Available studies have been highly biased, probably in favour of detecting an effect even if this is minimal, by their retrospective nature (missing for example, women who left the area before giving birth), not correcting for socioeconomic or ethic influences, using very raw and approximate models managing up to several square kilometres as identical areas of pollution, not compensating for time spent in traffic, at work or in other regions during weekends and holidays. Furthermore the discussion on the impact of air pollution had been partially taken over by politics, without robust data. Data from IPANEMA will help to estimate the real individual dose-effect relation for pregnant women.

Acknowledgements The authors thank Veronique De Vroeij and the midwifes of the Antwerp University Hospital for their help and support for the coordination of the study

Contributors LVdE and YJ have made substantial contributions to the conception and the design of the study and have been involved in drafting the manuscript. NL, $\mathrm{MB}, \mathrm{V}$ and GS have made substantial contributions to conception and design, or acquisition of data, or analysis and interpretation of data. VDV and MB have been involved in revising it critically for important intellectual content. All authors have given final approval of the version to be published.

Funding This research received no specific grant from any funding agency in the public, commercial or not-for-profit sectors.

Competing interests LVdE received a financial award from The Fondation Mustela. Roche Belgium will provide laboratory reagents, free of charge.

Patient consent Obtained.

Ethics approval Ethical Committee of the Antwerp University Hospital $(14 / 40 / 411)$

Provenance and peer review Not commissioned; externally peer reviewed.

Open Access This is an Open Access article distributed in accordance with the Creative Commons Attribution Non Commercial (CC BY-NC 4.0) license, which permits others to distribute, remix, adapt, build upon this work non-commercially, and license their derivative works on different terms, provided the original work is properly cited and the use is non-commercial. See: http://creativecommons.org/ licenses/by-nc/4.0/

(C) Article author(s) (or their employer(s) unless otherwise stated in the text of the article) 2018. All rights reserved. No commercial use is permitted unless otherwise expressly granted.

\section{REFERENCES}

1. Rudra CB, Williams MA, Sheppard L, et al. Ambient carbon monoxide and fine particulate matter in relation to preeclampsia and preterm delivery in western Washington State. Environ Health Perspect 2011;119:886-92.

2. Dadvand $P$, Figueras $F$, Basagaña $X$, et al. Ambient air pollution and preeclampsia: a spatiotemporal analysis. Environ Health Perspect 2013;121:1365-71.

3. Zhu X, Liu Y, Chen Y, et al. Maternal exposure to fine particulate matter (PM2.5) and pregnancy outcomes: a meta-analysis. Environ Sci Pollut Res 2015;22:3383-96.

4. Wu J, Ren C, Delfino RJ, et al. Association between local trafficgenerated air pollution and preeclampsia and preterm delivery in the south coast air basin of California. Environ Health Perspect 2009;117:1773-9. 
5. Yorifuji T, Naruse H, Kashima S, et al. Residential proximity to major roads and placenta/birth weight ratio. Sci Total Environ 2012;414:98-102.

6. WHO recommendations for Prevention and treatment of preeclampsia and eclampsia. Geneva, Switzerland: World Health Organization, 2011.

7. Steegers EA, von Dadelszen P, Duvekot JJ, et al. Pre-eclampsia. Lancet 2010;376:631-44.

8. Anderson UD, Olsson MG, Kristensen $\mathrm{KH}$, et al. Review: biochemical markers to predict preeclampsia. Placenta 2012;33:S42-S47.

9. Kaufmann P, Black S, Huppertz B. Endovascular trophoblast invasion: implications for the pathogenesis of intrauterine growth retardation and preeclampsia. Biol Reprod 2003;69:1-7.

10. Thomas CP, Andrews JI, Liu KZ. Intronic polyadenylation signal sequences and alternate splicing generate human soluble Flt1 variants and regulate the abundance of soluble Flt1 in the placenta. Faseb J 2007;21:3885-95.

11. Palei AC, Spradley FT, Warrington JP, et al. Pathophysiology of hypertension in pre-eclampsia: a lesson in integrative physiology. Acta Physiol 2013;208:224-33.

12. Li C, Raikwar NS, Santillan MK, et al. Aspirin inhibits expression of sFLT1 from human cytotrophoblasts induced by hypoxia, via cyclooxygenase 1. Placenta 2015;36:446-53.

13. Garcia-Tizon Larroca S, Tayyar A, Poon LC, et al. Competing risks model in screening for preeclampsia by biophysical and biochemical markers at 30-33 weeks' gestation. Fetal Diagn Ther 2014;36:9-17.

14. Stepan H, Herraiz I, Schlembach D, et al. Implementation of the sFIt-1/PIGF ratio for prediction and diagnosis of pre-eclampsia in singleton pregnancy: implications for clinical practice. Ultrasound Obstet Gynecol 2015;45:241-6.

15. Meler E, Scazzocchio E, Peguero A, et al. Role of maternal plasma levels of placental growth factor for the prediction of maternal complications in preeclampsia according to the gestational age at onset. Prenat Diagn 2014;34:n/a-10.

16. Padma Y, Aparna VB, Kalpana B, et al. Renal markers in normal and hypertensive disorders of pregnancy in Indian women: a pilot study. Int J Reprod Contraception. Obstet Gynecol 2013:514-20.

17. Saleh S, Antoniou A, Harrington K, et al. Second trimester maternal serum cystatin $C$ levels in preeclamptic and normotensive pregnancies: a small case-control study. Hypertens Pregnancy 2010;29:112-9.
18. Toda N, Nakanishi-Toda M. How mental stress affects endothelial function. Pflugers Arch 2011;462:779-94.

19. Zhang X, Staimer N, Tjoa T, et al. Associations between microvascular function and short-term exposure to traffic-related air pollution and particulate matter oxidative potential. Environ Health 2016;15:81.

20. Poursafa P, Baradaran-Mahdavi S, Moradi B, et al. The relationship of exposure to air pollutants in pregnancy with surrogate markers of endothelial dysfunction in umbilical cord. Environ Res 2016;146:154-60.

21. Zaichuk TA, Shroff EH, Emmanuel R, et al. Nuclear factor of activated $T$ cells balances angiogenesis activation and inhibition. J Exp Med 2004;199:1513-22.

22. Lee PC, Roberts JM, Catov JM, et al. First trimester exposure to ambient air pollution, pregnancy complications and adverse birth outcomes in Allegheny County, PA. Matern Child Health $J$ 2013;17:545-55.

23. Wilhelm M, Ritz B. Residential proximity to traffic and adverse birth outcomes in Los Angeles County, California, 1994-1996. Child Heal 2003:207-16.

24 Souza EV, Torloni MR, Atallah AN, et al. Aspirin plus calcium supplementation to prevent superimposed preeclampsia: a randomized trial. Braz J Med Biol Res 2014;47:419-25.

25. Kadmiel M, Cidlowski JA. Glucocorticoid receptor signaling in health and disease. Trends Pharmacol Sci 2013;34:518-30.

26. Stalder T, Kirschbaum C. Analysis of cortisol in hair--state of the art and future directions. Brain Behav Immun 2012;26:1019-29.

27. Braig S, Grabher F, Ntomchukwu C, et al. Determinants of maternal hair cortisol concentrations at delivery reflecting the last trimester of pregnancy. Psychoneuroendocrinology 2015;52:289-96.

28. Ohkuchi A, Hirashima $\mathrm{C}$, Suzuki $\mathrm{H}$, et al. Evaluation of a new and automated electrochemiluminescence immunoassay for plasma sFIt-1 and PIGF levels in women with preeclampsia. Hypertens Res 2010;33:422-7.

29. Schiettecatte J, Russcher H, Anckaert E, et al. Multicenter evaluation of the first automated Elecsys sFlt-1 and PIGF assays in normal pregnancies and preeclampsia. Clin Biochem 2010;43:768-70.

30. Grubb A, Blirup-Jensen S, Lindström V, et al. First certified reference material for cystatin $\mathrm{C}$ in human serum ERM-DA471/IFCC. Clin Chem Lab Med 2010;48:1619-21. 